\title{
Caracterização Estrutural de Polímeros e Associações Moleculares por Técnicas de Dispersão de Luz
}

\author{
NUNOC. SANTOS, MIGUELA.R.B. CASTANHO*
}

\section{INTRODUÇÃO}

O trabalho efectuado em 1869 , na Universidade de Cambridge, pelo meteorologista J. Tyndall, acerca da luz dispersa por aerossóis, pode ser considerado como a primeira tentativa científica para a explicação do clássico enigma "Porque é o céu azul?" [1,2]. Foi baseando-se na teoria electromagnética de J. C. Maxwell que, entre 1871 e 1918, J. W. Strutt (mais tarde Lord Rayleigh) desenvolveu um notável trabalho teórico, conseguindo explicar a dispersão de luz por pequenas partículas (tipicamente, inferiores a um vigésimo do comprimento de onda da luz), e consequentemente a cor azul do céu e o vermelho do pôr-do-sol [3-6] (acerca destes fenómenos naturais consultar e.g. [7]), desenvolvendo ainda uma fórmula para esferas de grandes dimensões. Esta teoria para esferas de dimensões arbitrárias, assim como partículas não esféricas, continuou a ser desenvolvida independentemente por Debye e Gans, nas décadas de 10 e 20, chegando-se assim à base teórica da Dispersão de Rayleigh (também por vezes designada por Rayleigh-Debye ou RayleighDebye-Gans). Independentemente dos trabalhos de Debye, em 1909 Mie desenvolveu um outro formalismo para o estudo de partículas de grandes dimensões (Dispersão de Mie). Nos anos 40, Debye, Zimm e colaboradores desenvolveram a dispersão de luz como um método para a determinação de massas moleculares, dimensões, formas e interacções entre macromoléculas ou agregados supramoleculares em solução. Estes estudos, lidando apenas com a intensidade da luz dispersa, deram origem aos formalismos e técnica denominada Dispersão estática de luz, SLS (static light scattering), ILS (intensity light scattering) ou Dispersão de luz clássica. Para revisão ver e.g. [8,9].

Um desenvolvimento paralelo para as técnicas de dispersão de luz nasceu em 1914 com o trabalho de L. Brillouin, ao prever teoricamente o aparecimento de um dupleto de picos no espectro de dispersão, moti- vado por ondas sonoras. Experiências realizadas por Gross, nos anos 30 , comprovaram experimentalmente a existência destes dois picos (dupleto de Brillouin) com desvio idêntico a um pico central de luz dispersa com frequência média igual à da radiação incidente, denominado pico de Rayleigh. Uma terceira classe de radiação dispersa foi verificada pela primeira vez em 1927 por Raman. Todos estes estudos de radiação dispersa a frequências diferentes da radiação incidente levaram, mais tarde, ao aparecimento da Dispersão dinâmica de luz, DLS (dynamic light scattering), (ou Dispersão quase elástica de luz, QELS quasi-elastic light scattering, ou Espectroscopia de correlação fotónica, PCS Photon correlation spectroscopy), bem como da Dispersão de Brillouin e Dispersão de Raman.

Em 1934, Landau e Placzek, conseguiram explicar teoricamente, com base na termodinâmica, o pico de Rayleigh. Contudo, o desenvolvimento da Dispersão dinâmica de luz foi impedido pela impossibilidade da obtenção de uma radiação incidente suficientemente monocromática, limitando-se a sua utilização a um número bastante reduzido de investigadores, especialmente na então União Soviética e na Índia. Esta situação só foi alterada no início da década de 60 com o desenvolvimento das fontes laser, as quais vieram derrubar esta limitação experimental. R. Pecora, na sua tese de doutoramento, em 1962. assim como em trabalhos posteriores, desenvolveu os fundamentos teóricos para a evolução da técnica, sendo Cummins, Knable e Yeh, em 1964, os pioneiros da sua aplicação experimental [10], abrindo um vasto leque de possíveis utilizações $[9,11]$. Além de quase monocromática a luz laser é produzida com grande intensidade, o que é bastante importante atendendo ao facto de apenas uma ínfima fracção do total da luz incidente sofrer dispersão.

Em grande parte devido às suas possibilidades na determinação de dimensões (DLS e SLS), formas (conjugação de ambas as técnicas), coeficientes de difusão (DLS) e massas mo- leculares (SLS), não causando a destruição ou alteração da amostra, as técnicas espectroscópicas de dispersão de luz foram implantadas na comunidade científica no decorrer da década de 70 . A sua aplicação a novos problemas (e.g., conformação de polímeros e agregação de surfactantes), aliada a desenvolvimentos tecnológicos, levaram à sua optimização, assim como ao nascer de inúmeras variantes, incluindo o acoplamento a diferentes técnicas (e.g. [1218]). Assim, a investigação através de técnicas de Dispersão de Luz é, actualmente, um campo de investigação de grande importância na Química, Física, Bioquímica e Biologia (para revisão mais aprofundada consultar e.g. [19-22]).

\section{ASPECTOS GERAIS}

A radiação electromagnética, ao incidir na matéria, pode não interactuar com esta, sendo transmitida; pode igualmente ser absorvida, provocando uma reacção química, aquecimento, ou a sua emissão por fluorescência ou fosforescência. Para além destas possibilidades, a radiação pode igualmente ser dispersa.

O fenómeno da dispersão de luz ocorre quando o campo eléctrico da radiação incidente num átomo induz oscilações periódicas na sua nuvem electrónica, passando deste modo a funcionar como fonte secundária de radiação. Na dispersão de Rayleigh, a componente eléctrica do campo electromagnético da radiação incidente

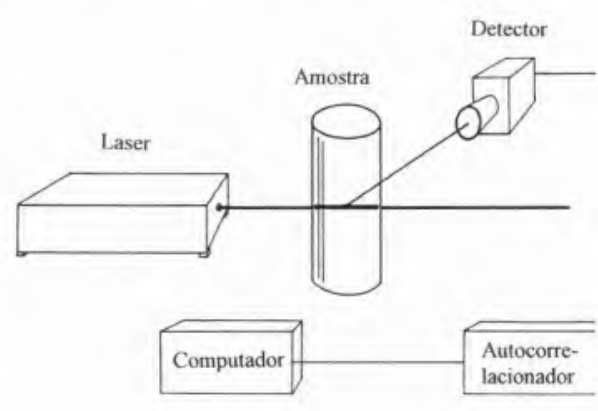

Fig. 1 - Representação esquemática de uma experiência de dispersão estática de luz. 
faz com que os electrões oscilem sobre a sua posição de equilíbrio, criando um dipolo oscilante. Este funciona como uma antena, reemitindo a radiação com a mesma frequência. É esta a radiação dita dispersa.

\section{INSTRUMENTAÇÃO}

O equipamento necessário para medidas de dispersão de luz é constituído pelo laser, porta-células, detector, goniómetro, autocorrelacionador e computador (Fig. 1). Os lasers mais utilizados são os de $\mathrm{He}-\mathrm{Ne}, \mathrm{Ar}^{+}$e $\mathrm{Kr}^{+}$ (antes do seu aparecimento eram utilizadas lâmpadas de mercúrio). A sua luz é focada na amostra, preparada numa célula de dispersão de luz. Esta fica contida no porta-células, imersa num banho termostatizado de um composto com índice de refracção semelhante ao do vidro, usualmente decalina (decahidronaftaleno), evitando a ocorrência de reflexões. O detector, montado sobre o goniómetro, permite efectuar as leituras a diferentes ângulos de dispersão ${ }^{1}(\theta)$. O detector pode ser precedido por um polarizador, de modo a permitir analisar apenas a radiação com uma dada polarização (medidas de anisotropia de dispersão de luz). A intercepção do feixe incidente com o feixe captado pelo detector, define $o$ volume de dispersão (V), que atinge o seu valor mínimo para $\theta=90^{\circ}$. Os valores obtidos pelo detector seguem para o autocorrelacionador (só necessário para medidas de Dispersão dinâmica de luz) e deste para um computador, onde é efectuado o tratamento dos dados $[9,23]$.

\section{PREPARAÇÃO DAS AMOSTRAS}

Qualquer partícula contaminante de dimensões superiores às das partículas em estudo (genericamente designadas por poeiras) pode eventualmente causar uma dispersão várias ordens de grandeza superior à destas. Portanto, todo o processo de preparação das amostras deve ser orientado no sentido de evitar a sua presença. Assim, a amostra deve ser cuidadosamente filtrada através de uma membrana de dimensão de poro adequada, para uma célula previamente lavada com mistura cromosulfúrica (por ex.) e abundantemente lavada com água destilada filtrada. Caso seja necessário a amostra deve ainda ser centrifugada.

\section{DISPERSÃO ESTÁTICA DE LUZ}

A expressão obtida por Rayleigh para a determinação da intensidade de luz dispersa por uma partícula pontual isolada é,

$$
\frac{I_{\mathrm{s}}}{I_{0}}=\frac{16 \pi^{4} \alpha^{2} \operatorname{sen}^{2} \theta_{z}}{r^{2} \lambda^{4}}
$$

onde $\mathrm{I}_{\mathrm{s}}$ é a intensidade de luz dispersa, $\mathrm{I}_{\mathrm{O}}$ a intensidade de luz incidente, polarizada verticalmente, $\alpha$ a polarizabilidade da partícula, $\theta_{2}$ o ângulo de observação relativamente ao eixo vertical (o da polarização da luz incidente), $r$ a distância ao detector, e $\lambda$ o comprimento de onda da radiação incidente. É o facto de $\mathrm{I}_{\mathrm{S}}$ ser inversamente proporcional à quarta potência de $\lambda$ que explica o azul do céu e o vermelho do pôr-do-sol. Assim, visto a radiação azul proveniente do sol ser predominantemente dispersa, é esta que se observa preferencialmente quando se olha o céu a um ângulo afastado da fonte de radiação (sol). Pelo contrário, ao olharmos directamente o sol nascente ou poente, é a radiação vermelha que observamos, uma vez que esta é a que menos sofre dispersão ao atravessar a atmosfera terrestre (e.g. [24]).

De modo a eliminar o factor geométrico $\mathrm{r}^{2}$ e o $\operatorname{sen} \theta_{7}$ (se a medida não for feita no plano perpendicular à polarização da radiação incidente). na prática utiliza-se a razão de Rayleigh, definida pela equação,

$$
\mathrm{R}_{\theta}=\frac{\mathrm{r}^{2}}{\operatorname{sen} \theta_{\mathrm{z}}} \frac{\mathrm{I}_{\mathrm{s}}}{\mathrm{I}_{\mathrm{o}}}=\frac{16 \pi^{4} \alpha^{2}}{\lambda^{4}}
$$

Como se pode observar, $R_{\theta}$ é proporcional a $\mathrm{I}_{\mathrm{s}}$, dependendo a constante de proporcionalidade apenas de factores instrumentais.

Para uma solução ideal de N partículas dispersantes independentes por unidade de volume, admitindo que estas são pequenas comparadas $\operatorname{com} \lambda$ (tipicamente $<\lambda / 20$ ), teremos,

$$
\mathrm{R}_{\theta}=\mathrm{N} \frac{16 \pi^{4} \alpha^{2}}{\lambda^{4}}
$$

Sendo,

$$
\begin{aligned}
& \alpha=\frac{\mathrm{n}_{\mathrm{o}}}{2_{\pi}} \frac{\mathrm{dn}}{\mathrm{dc}} \frac{\mathrm{M}_{\mathrm{w}}}{\mathrm{N}_{\mathrm{A}}} \\
& \mathrm{N}=\frac{\mathrm{cN}_{\mathrm{A}}}{\mathrm{M}_{\mathrm{W}}}
\end{aligned}
$$

obtemos,

$$
R_{\theta}=\frac{4 \pi^{2} n_{0}^{2}\left(\frac{d n}{d c}\right)^{2}}{N_{A} \lambda^{4}} M_{W} c=K M_{W} c
$$

onde $\mathrm{n}_{0}$ é o índice de refracção do solvente, $(\mathrm{dn} / \mathrm{dc})$ o incremento do índice de refracção específico da solução, $M_{w}$ a massa molar do dispersante, c a sua concentração (em massa), e $\mathrm{N}_{\mathrm{A}}$ a constante de Avogadro. A constante $K$,que vem simplificar

$$
\mathrm{K}=\frac{4 \pi^{2} \mathrm{n}_{\mathrm{o}}^{2}\left(\frac{\mathrm{dn}}{\mathrm{dc}}\right)^{2}}{\mathrm{~N}_{\mathrm{A}} \lambda^{4}}
$$

a expressão, é designada por constante óptica do sistema soluto/solvente em estudo (e.g. [25]). Esta equação só pode ser usada para sistemas diluídos, onde é válida a aproximação das partículas dispersantes serem independentes, sendo nestes casos possível a determinação da massa molar da partícula através da equação (em que se admite $\mathrm{dn} / \mathrm{dc}$ constante, uma aproximação válida na generalidade dos casos),

$$
\frac{\mathrm{Kc}}{\mathrm{R}_{\theta}}=\frac{1}{\mathrm{M}_{\mathrm{w}}}
$$


No caso de um sistema não ideal de partículas pequenas a equação tem que ser alterada através da introdução do segundo coeficiente do viri$\mathrm{al}^{2}\left(\mathrm{~A}_{2}\right)$, de modo a prever as interacções soluto/soluto e soluto/solvente,

$$
\frac{\mathrm{KC}}{\mathrm{R}_{\theta}}=\frac{1}{\mathrm{M}_{\mathrm{w}}}+2 \mathrm{~A}_{2} \mathrm{C}
$$

Para partículas grandes comparadas com o comprimento de onda da radiação incidente $(>\lambda / 20)$ os dipolos podem ser induzidos em diferentes partes da macromolécula pela radiação incidente, causando interferências destrutivas aquando da reemissão de luz pela diferentes partes da molécula. A interferência destrutiva será maior a ângulos maiores no plano $x y$, relativamente ao feixe transmitido (Fig. 2) [24]. Nesta situação torna-se útil definir o factor pelo qual a intensidade é reduzida a um dado ângulo, $\theta$,

$\mathrm{P}_{\theta}=\frac{\mathrm{I}_{\theta}}{\mathrm{I}_{\theta}^{\mathrm{o}}}=1-\frac{\mathrm{q}^{2} \mathrm{R}_{\mathrm{g}}^{2}}{3}+\ldots$

onde $\mathrm{I}_{\theta}$ é a intensidade de luz dispersa ao ângulo de observação, $\mathrm{R}_{\mathrm{g}}$ é o raio de giração e q o vector de dispersão, definido por,

$$
\mathrm{q}=\frac{4 \pi \mathrm{n}_{0}}{\lambda} \operatorname{sen}\left(\frac{\theta}{2}\right)
$$

[26]. Portanto, para um sistema não ideal de partículas grandes podemos utilizar a expressão,

$\frac{K c}{R_{\theta}}=\left(\frac{1}{M_{w}}+2 A_{2} C\right) \frac{1}{P_{\theta}}$

(sendo o parâmetro $\mathrm{P}_{\theta^{-1}}$ designado factor geométrico estático), ou, numa forma mais extensa e aproximada ${ }^{3}$ (combinando as eq. 10, 11 e 12),

$$
\begin{gathered}
\frac{K c}{R_{\theta}}=\left(\frac{1}{M_{w}}+2 A_{2} C\right) \\
\left(1+\frac{16 \pi^{2} n_{o}^{2} R_{g}^{2}}{3 \lambda^{2}} \operatorname{sen}^{2}\left(\frac{\theta}{2}\right)\right)
\end{gathered}
$$

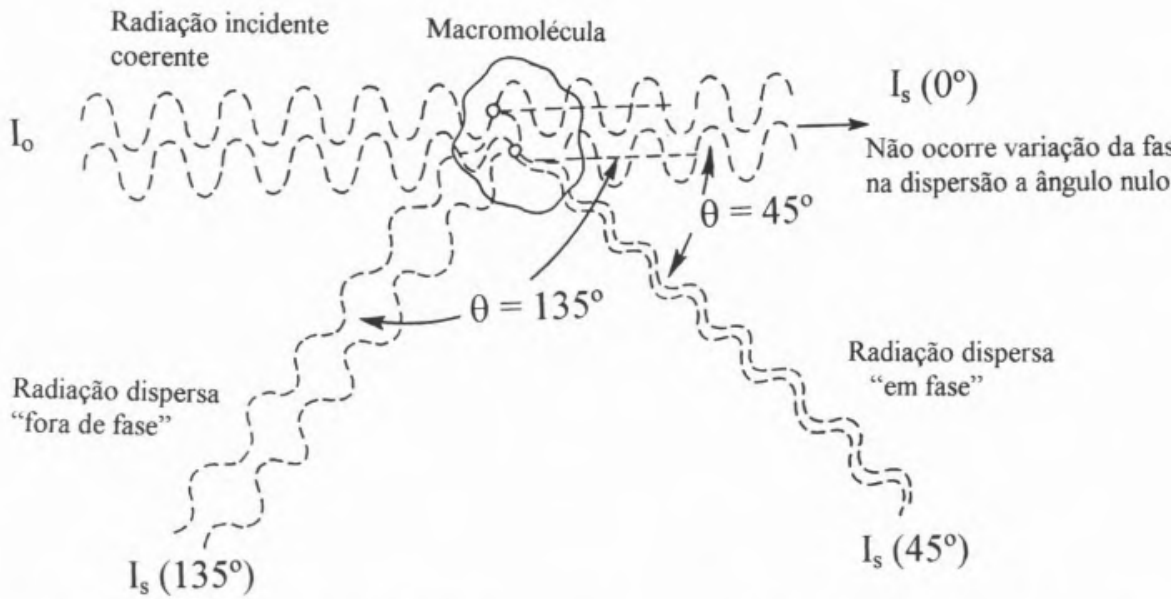

Fig. 2 - Relação de fase entre a radiação incidente (coerente), e a dispersa a vários ângulos por dois electrões da mesma macromolécula.

Se estiver em estudo uma amostra polidispersa, $\mathrm{M}_{\mathrm{w}}$ é a massa molar média ponderada pelas massas,

$$
M_{w}=\frac{\sum w_{i} M_{i}}{\sum w_{i}}
$$

verificando-se o mesmo para $\mathrm{R}_{\mathrm{g}}{ }^{2}$,

$$
R_{g}=\sqrt{\frac{\sum w_{i} R_{g, i}{ }^{2}}{\sum w_{i}}}
$$

Pelo contrário, o valor obtido para o segundo coeficiente do virial tem uma ponderação mais complexa,

$$
A_{2}=\frac{\sum w_{i}^{2} M_{i} A_{2, i}}{\left(\sum w_{i}\right)\left(\sum w_{i} M_{i}\right)}
$$

O raio de giração é calculado sem se pressupor qualquer geometria para a partícula. Na Tabela 1, podemos observar a relação entre $o R_{g}$ e as dimensões características de várias geometrias [27].

\section{MÉTODO DE ZIMM}

A obtenção dos parâmetros $R_{\mathrm{g}}$, $\mathrm{M}_{\mathrm{w}}$ e $\mathrm{A}_{2}$ é usualmente feita recorrendo ao método de Zimm [24,25]. Para a sua aplicação é necessário medir a intensidade da dispersão de luz a diferentes ângulos, para cada uma das concentrações estudadas. Cada um dos valores obtidos tem que ser corrigido, através da subtracção do valor obtido para a intensidade de dispersão do solvente para o mesmo ângulo de dispersão. O método de Zimm baseia-se na eq. 13, recorrendo à representação de $\mathrm{K}_{\mathrm{c}} / \mathrm{R}_{\theta}$ em função de $\operatorname{sen}^{2}(\theta / 2)+k c$ (Fig. 3). $\mathrm{O}$ parâmetro k é uma constante arbitrária, sendo somente utilizado para facilitar a interpretação da representação gráfica. A conversão de $\mathrm{I}_{\mathrm{s}} \mathrm{em}$ $R_{\theta}$ é feita recorrendo a um padrão (e.g. benzeno),

$\mathrm{R}_{\theta}=\mathrm{R}_{\text {benzeno, } 90^{\circ}} \frac{\mathrm{n}_{\mathrm{o}}^{2}}{\mathrm{n}_{\text {benzeno }}{ }^{2}} \frac{\mathrm{I}_{\mathrm{s}} \operatorname{sen} \theta}{\mathrm{I}_{\text {benzeno }}}$

( $\mathrm{R}_{\text {benzeno.90 }}{ }^{\circ}$ é um valor previamente conhecido).

Extrapolando para ângulo zero as medidas efectuadas às várias concentrações (Fig. 3), é esperada uma relação linear entre os pontos extrapolados. Do declive da recta obtida destes pontos $\left(2 \mathrm{~A}_{2}\right)$ tiramos o valor de $\mathrm{A}_{2}$. Procedendo de forma inversa, extrapolando para concentração zero os pontos correspondentes aos vários ângulos de medida, a recta obtida dos pontos correspondentes à extrapolação para cada ângulo tem declive 
Tabela 1 - Correspondência entre o quadrado do raio de giração $\left(R_{\mathrm{g}}{ }^{2}\right)$ e as dimensões características de cada geometria da macromolécula.

\begin{tabular}{ll}
\hline Esfera de raio $R$ & $\frac{3}{5} R^{2}$ \\
\hline
\end{tabular}

Superfície esférica de raio externo $R$ e raio interno $c R(c>1) \quad \frac{3}{5}\left(c^{2}+\frac{c+1}{c^{2}+c+1}\right) R^{2}$

Superfície esférica infinitamente fina de raio $\mathrm{R}$

$\mathrm{R}^{2}$

Cilindro de comprimento $2 R$ e razão axial p $\quad \frac{1}{12}\left(4+\frac{3}{p^{2}}\right) R^{2}$

\begin{tabular}{ll} 
Cilindro infinitamente de comprimento $2 R$ & $\frac{1}{3} R^{2}$ \\
\hline Disco circular infinitamente fino de raio $R$ & $\frac{1}{2} R^{2}$ \\
\hline Elipsoide de revolução de eixo maior 2R e razão axial $p$ & $\frac{1}{5}\left(2+p^{2}\right) R^{2}$
\end{tabular}

\begin{tabular}{ll}
\hline Novelo aleatório de distância média entre os extremos $R$ & $\frac{1}{6} R^{2}$ \\
\hline Distribuição Gaussiana (exp (-aR2)) & $\frac{3}{2}$ a \\
\hline
\end{tabular}

$\frac{16 \pi^{2} n_{0}{ }^{2} R^{2}}{3 \lambda^{2}} \frac{1}{M_{w}}$, de onde se pode tirar o valor de $\mathrm{R}_{\mathrm{g}}$. O valor de $\mathrm{M}_{\mathrm{w}}$ é calculado a partir da ordenada na origem das rectas obtidas pela extrapolação da concentração para zero e da extrapolação do ângulo de dispersão para zero $\left(1 / \mathrm{M}_{\mathrm{w}}\right)$, devendo ambas coincidir no mesmo ponto.

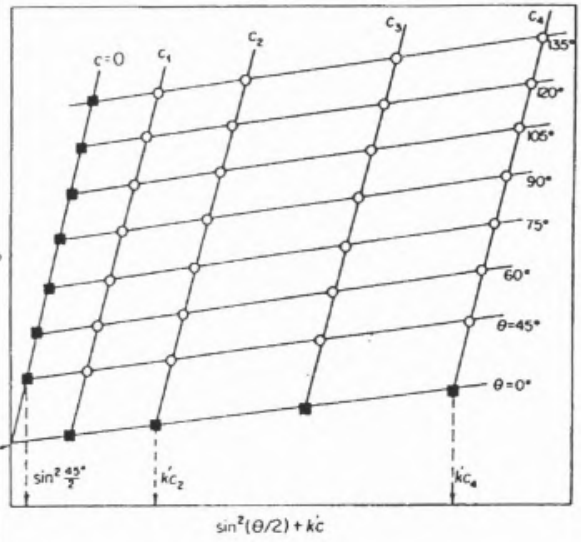

Fig. 3 - Exemplo de representação recorrendo ao Gráfico de Zimm. ticos internos da molécula dispersante (que pode absorver ou ceder alguma energia ao fotão), está fora do âmbito deste trabalho, assim como as linhas de Brillouin. O alargamento do pico de Rayleigh devido ao deslocamento do dispersante relativamente ao observador (efeito de Doppler) serve como fundamento para os estudos por dispersão dinâmica de luz.

As técnicas de dispersão dinâmica de luz baseiam-se nas flutuações das concentrações locais, devidas aos movimentos Brownianos das partículas, não existindo, até ao advento destas técnicas, qualquer forma de os medir. A dispersão de luz dinâmica baseia-se no conhecido efeito de Doppler, segundo o qual a frequência da radiação emitida por um ponto em movimento sofre um desvio para frequências superiores ou inferiores, consoante este se aproxima ou afasta do observador. Este desvio é função da velocidade do emissor.

Contudo, para macromoléculas, devido à sua baixa velocidade, este alargamento da banda em frequências $(\omega)$, descrito pela função $S(\omega)$ (eq. 18), é bastante pequeno, sendo impossível a sua resolução através de um interferómetro. Este impedimento é contornado pelo recurso à transformada de Fourier e a utilização de processadores matemáticos rápidos

\section{da à mesma frequência da radiação incidente, a radiação dispersa pode sofrer desvios relativamente a esta (Fig. 4). A dispersão de Raman, de- vida a alterações nos estados energé- \\ DISPERSÃO DINÂMICA DE LUZ}

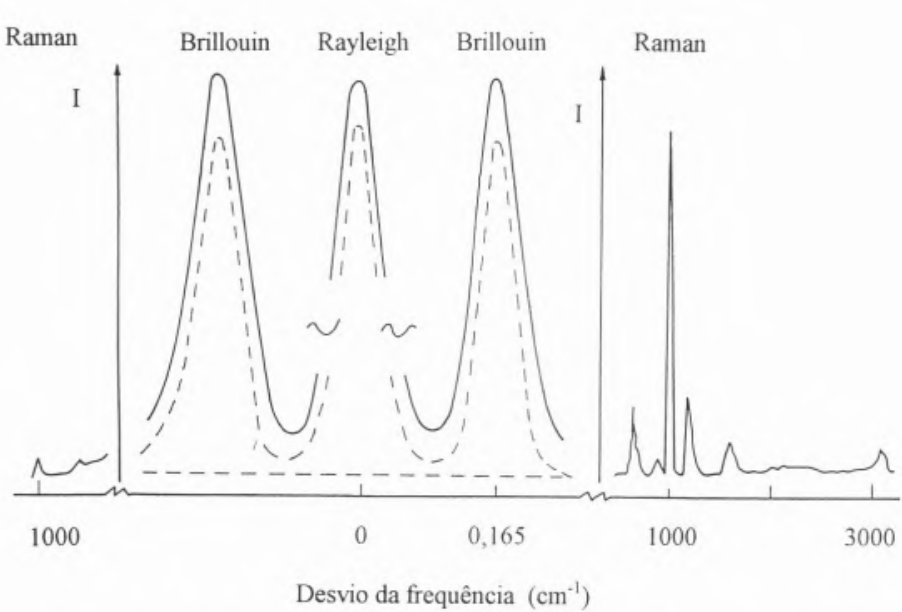

Fig. 4 - Esboço do espectro de dispersão do benzeno, mostrando as posições relativas das linhas de Rayleigh, Brillouin e Raman. 


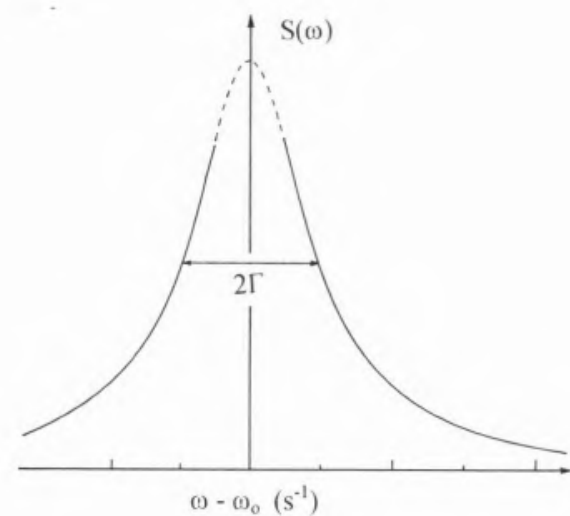

Fig. 5 - Correspondência entre as funções $S(\omega)$ e $C(t)$.

(autocorrelacionadores), que permitem o cálculo de uma função temporal, $\mathrm{C}(\mathrm{t})$, que está relacionada com $\mathrm{S}(\omega)$ do modo descrito pelas equações 18 e 19 [28].

$$
\begin{aligned}
& S(\omega)=\frac{1}{2 \pi} \int_{-\infty}^{+\infty} C(t) e^{-i(\omega t)} d t \\
& C(t)=\int_{-\infty}^{+\infty} S(\omega) e^{-i(\omega t)} d \omega
\end{aligned}
$$

Nestas funções $t$ não representa um tempo de vida mas sim o inverso de uma frequência. $\Gamma$ representa o inverso do tempo de decaimento característico, definido como o

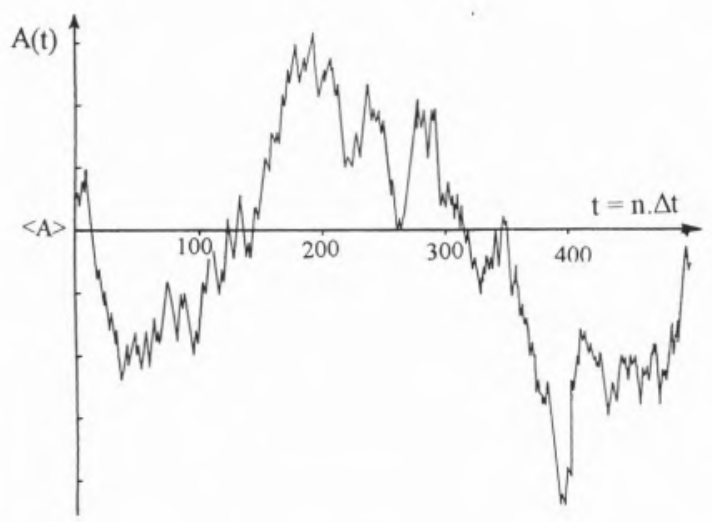

Fig. 6 - Representação esquemática das grandes flutuações da intensidade de dispersão obtida em medições de DLS.

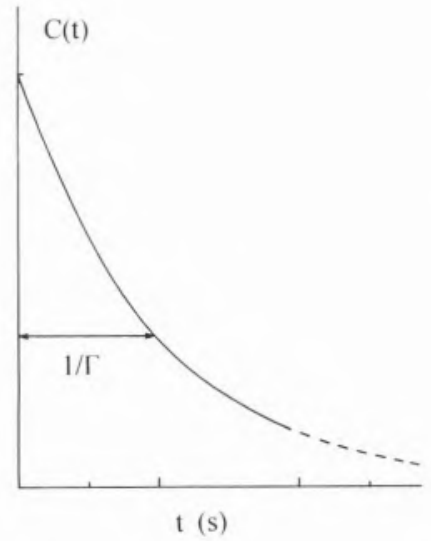

tempo necessário para que se dê um decréscimo a l/e do valor inicial (Fig. 5).

Ao contrário do SLS, que utiliza volumes de dispersão e tempos de registo elevados (mesmo da ordem dos $\mu$ s), em DLS utilizam-se volumes de dispersão bastante reduzidos, e tempos de análise da ordem dos $\mu$ s. Assim, podem detectar-se grandes flutuações na intensidade de luz dispersa, registadas pelo autocorrelacionador (Fig. 6). Sendo $A_{i}$ o número de fotões que chegam ao detector num intervalo de tempo $\Delta \mathrm{t}$, o autocorrelacionador multiplica este valor pelo do intervalo seguinte, registando o valor no canal 1 . O procedimento é repetido cerca de $10^{5}$ vezes, acumulando-se os resultados no mesmo canal. No canal 2 são registados os produtos de $A_{i}$ por $A_{i+2}$, continuando até ao n-ésimo canal. Obtém-se assim a função de correlação temporal, $\mathrm{G}_{2}(\mathrm{t})$,

$\mathrm{G}_{2}(\mathrm{t})=\mathrm{I}(\mathrm{t}) \times \mathrm{I}(\mathrm{t}+\Delta \mathrm{t})$

(I(t) é o número de fotões registados no canal correspondente ao tempo t). Se forem utilizados valores de $\Delta \mathrm{t}$ reduzidos, no intervalo de tempo correspondente aos primeiros canais as moléculas ainda não terão colidido com outras, permanecendo as velocidades e trajectórias do seu movimento semelhantes; teremos assim, nestes canais, um produto próximo de $\left\langle\mathrm{I}^{2}\right\rangle$ (Fig. 7). Para tempos muito longos a molécula terá sofrido várias colisões, perdendo qualquer correlação com a situação inicial; teremos agora um produto próximo de $<\mathrm{I}>2$ (Fig. 7), correspondendo ao valor obtido por SLS.

A função de correlação, normalizada a tempo infinito pela divisão por $<\mathrm{I}>^{2}\left(\mathrm{~g}_{2}\right)$, está relacionada com a função de correlação normalizada do campo eléctrico disperso $\left(\mathrm{g}_{1}\right)$ pela relação de Siegert,

$$
\mathrm{g}_{2}(\mathrm{t})=1+\beta\left(\mathrm{g}_{1}(\mathrm{t})\right)^{2}
$$

A presença de $\beta$ permite prever os desvios relativamente a uma correlação ideal. Nessa situação o parâmetro tomaria o valor 1 [29].

Para uma distribuição monodispersa de partículas pequenas comparativamente $\operatorname{com} \lambda$, ou para esferas de qualquer dimensão, obtém-se a equação,

$$
\mathrm{g}_{1}(\mathrm{t})=\mathrm{e}-\Gamma \mathrm{t}=\mathrm{e}^{-D q^{2}} \mathrm{t}
$$

onde D é o coeficiente de difusão da partícula. Para esferas pequenas, não interactuando entre si, numa solução diluída, podemos obter o raio hidrodinâmico $\left(\mathrm{R}_{\mathrm{h}}\right)$ da partícula, utilizando a equação de Stokes-Einstein,

$$
\mathrm{D}_{\mathrm{o}}=\frac{\mathrm{kT}}{6 \pi \eta_{\mathrm{o}} \mathrm{R}_{\mathrm{h}}}
$$

onde $\mathrm{k}$ representa a constante de Boltzman, $\mathrm{T}$ a temperatura absoluta, e $\eta_{0}$ a viscosidade do solvente. Visto D poder variar consideravelmente com a concentração, torna-se necessária a utilização do coeficiente de difusão a diluição infinita $\left(\mathrm{D}_{\mathrm{o}}\right)$ [30].

\section{OBTENÇÃO DE $\mathbf{D}_{\mathrm{o}}$ e $\mathbf{R}_{\mathrm{h}}$}

Podem surgir dificuldades na análise dos resultados de DLS se a amostra for significativamente polidispersa [31]. Se o sistema contém partículas com uma gama larga de dimensões, formando uma distribuição contínua, então poderemos escrever, 
$<\mathrm{I}(\mathrm{t}) \cdot \mathrm{I}(\mathrm{t}+\Delta \mathrm{t})>$

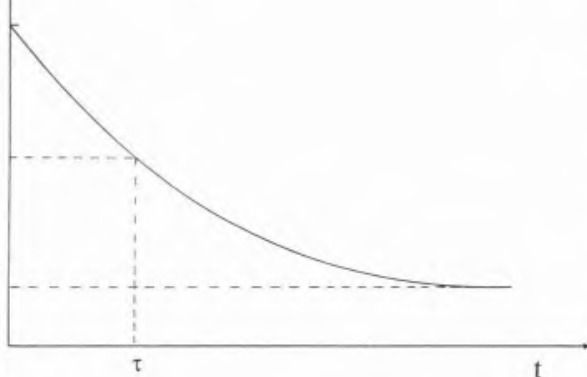

Fig. 7 - Função de correlação temporal. $\tau$ representa o tempo de relaxação ou de correlação, definido como o tempo necessário para que o valor da função de correlação decresça a 1/e do seu valor inicial.

$$
g_{1}(t)=\int_{-\infty}^{+\infty} G(\Gamma) e^{-i(\Gamma t)} d \Gamma
$$

onde $G(\Gamma)$ representa a distribuição de intensidades normalizada do decaimento. Como se pode verificar, $\mathrm{g}_{1}(\mathrm{t})$ é a transformada de Laplace de $\mathrm{G}(\Gamma)$ em relação a $\Gamma$. Assim, é do máximo interesse efectuar a inversão da transformada de Laplace, de modo a obter a distribuição dos vários componentes da função de correlação temporal. Contudo, para isto ser possível de forma fiável, são necessários resultados com pouco ruído (grande número de contagens por canal). O método mais utilizado para este fim é denominado CONTIN. Este método foi desenvolvido por Provencher em 1982, e está disponível em software comercial. A análise decorre em dois ciclos, sendo o primeiro uma análise superficial da informação de modo a escolher um conjunto inicial de parâmetros, e o segundo a determinação do conjunto de parâmetros que melhor se adaptam aos resultados experimentais, obtendo-se no final informação numérica e o histograma da distribuição de tamanhos [32]. Na Fig. 8 podemos observar um exemplo de histograma de distribuição de dimensões obtido pelo método CONTIN. Para uma comparação destes e de outros métodos consultar e.g. $[33,34]$.

Se, no entanto, os resultados iniciais não apresentarem baixo nível de ruído, o método facilmente gera soluções (histogramas) sem significado físico. O uso do CONTIN torna-se impossível. Pode usar-se então o método dos cumulantes, que nos fornece informação estatística sobre a distribuição, mas não a distribuição em si. Este método baseia-se no estudo da função de correlação temporal a tempos reduzidos, onde a sua parte inicial pode ser aproximada por uma única função exponencial. Utiliza-se então a chamada expansão de cumulantes, onde o logaritmo da função é expandido numa série de potências, obtendo-se,

$\ln \left(g_{1}(t)\right)=-\Gamma_{1} t+\frac{\Gamma_{2}}{2} t^{2}-\frac{\Gamma_{3}}{3} t^{3}+\ldots$

onde $\Gamma_{\mathrm{i}}$ representa o i-ésimo cumulante. Do primeiro cumulante obtém-se o coeficiente de difusão médio do dispersante à concentração c, $\mathrm{D}_{\mathrm{c}}$,

$$
\Gamma_{1}=D_{C} q^{2}\left(1+C R_{g}^{2} q^{2}\right)
$$

onde q é o vector de dispersão, $\mathrm{R}_{\mathrm{g}}$ é o raio de giração, e C é um factor característico da geometria da molécula, sendo nulo para esferas rígidas [35]. $D_{c}$ está relacionado com $D_{0}$

$$
D_{c}=D_{o}\left(1+k_{D} C+\ldots\right)
$$

por, onde $\mathrm{k}_{\mathrm{D}}$ é uma constante.

De modo a evitar incorrecções nos resultados e a obter o valor de $\mathrm{D}_{\mathrm{o}}$, o estudo deve ser efectuado para várias concentrações e a diferentes ângulos de dispersão $(\theta)$. O valor de $D_{0}$ é obtido através da extrapolação dos diferentes valores de coeficientes de difusão obtidos para concentração nula e $\operatorname{sen}^{2}(\theta / 2)$ $=0$, de forma análoga ao método de Zimm, utilizado em SLS. O raio hidrodinâmico $\left(R_{h}\right)$ das partículas dispersantes é depois obtido pela equação de Stokes-Einstein (eq. 23).

\section{GEOMETRIA DAS PARTÍCULAS - O FACTOR $\mathbf{R}_{\mathrm{g}} / \mathbf{R}_{\mathrm{h}}$}

O parâmetro $\rho$, definido como a razão $R_{g} / R_{h}$, tem sido utilizado como indicador da geometria das partículas dispersantes, uma vez que varia bastante com a compacticidade ou "alongamento" destas. A forma mais compacta possível de uma partícula é a esférica. Neste caso $R_{g}$ é mínimo. Se a esfera for alongada segundo um eixo, aproxi-

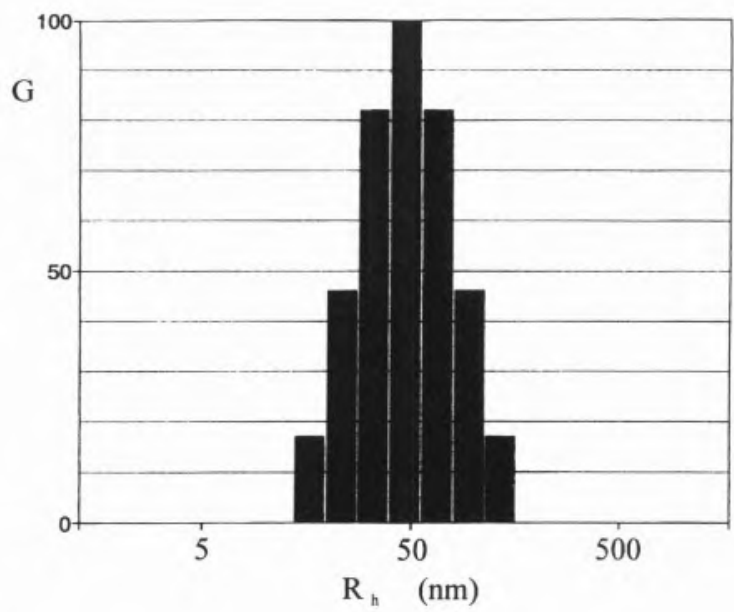

Fig. 8 - Histograma da distribuição de dimensões, obtido pelo método CONTIN.

mando-se de um elipsóide, $\mathrm{R}_{\mathrm{g}}$ aumenta sem que $\mathrm{R}_{\mathrm{h}}$ aumente correspondentemente, conduzindo a um aumento de $\rho$. O caso extremo é o de um cilindro fino e longo, em que $R_{g} / R_{h}$ atinge os valores máximos. Para uma esfera de densidade homogénea $\rho=0,775$, enquanto no caso de um cilindro cujo comprimento é muito superior ao diâmetro $\rho \geq 2$ (Tabela 2) [36,37]. As partículas globulares são casos intermédios. 
Tabela 2 - Valores de $\rho\left(R_{p} / R_{h}\right)$ para diferentes geometrias de partículas dispersantes. $\mathrm{f}$ - funcionalidade do polímero (número de ramificações). $\mathrm{z} \sim \beta \mathrm{N}^{1 / 2}$, onde $\beta$ representa o volume excluído de um segmento e $\mathrm{N}$ o número de segmentos

\begin{tabular}{ll}
\hline Geometria & $\boldsymbol{\rho}$ \\
\hline Esfera homogénea & 0,778 \\
Novelo aleatório, monodisperso & \\
$\quad$ Condições $\Theta$ & 1,50 \\
$\quad$ Bom solvente & 1,78 \\
Novelo aleatório, polidisperso, $\mathrm{z}=1$ \\
$\quad$ Condições $\Theta$ \\
$\quad$ Bom solvente & 1,73 \\
Estrela Regular & 2,05 \\
$\quad$ Condições $\Theta, f=4$ & \\
$\quad$ Condições $\Theta, f>>1$ & 1,33 \\
Cilindro rígido & 1,08 \\
$\quad$ Monodisperso & \\
$\quad$ Polidisperso, $\mathrm{z}=1$ & $>2,0$ \\
\hline
\end{tabular}

\section{CONCLUSÕES}

As técnicas espectroscópicas de dispersão de luz constituem um meio simples e eficaz de calcular a massa molar, dimensões $\left(R_{g}\right.$ e $\left.R_{h}\right)$ e $\mathrm{A}_{2}$ de um polímero ou agregado molecular. A conjugação de dados de dispersão estática e dinâmica de luz permite ainda concluir sobre a sua geometria. Tem ainda a grande vantagem de ser um processo não destrutivo.

O estudo é efectuado sem estar sujeito a artefactos causados pelo isolamento da amostra, secagem ou perda de matéria durante os processos de preparação ou observação. Actualmente estas técnicas são largamente aplicadas no estudo estrutural de soluções de macromoléculas, incluindo polímeros sintéticos, proteínas, polissacáridos, vírus, micelas, partículas e agregados coloidais, e microemulsões.

\footnotetext{
* A quem a correspondência deve ser dirigida: Centro de Química Física Molecular. Complexo I, I. S. T. - 1096 - Lisboa codex, Portugal Departamento de Química e Bioquímica, Faculdade de Ciências da Universidade de Lisboa, 1700 - Lisboa, Portugal
}

\section{NOTAS}

1 Alternativamente pode ser utilizado um detector fixo, ao qual se liga uma fibra óptica, cujo extremo oposto recolhe a luz dispersa a um ângulo regulável.

2 A utilizaçāo do termo linear é, na maior parte dos casos, suficiente.

3 Para pequenos valores de $\mathrm{x}, \frac{1}{1-\mathrm{x}} \sim 1+\mathrm{x}$.

\section{REFERÊNCIAS}

1. Tyndall, J. (1869) Phil. Mag., 37, 384-394.

2. Tyndall, I. (1869) Phil. Mag., 38, 156-158.

3. Strutt, I.W. (1871) Phil. Mag., 41, 107-120.

4. Strutt, J.W. (1871) Phil, Mag., 41, 274-279.

5. Strutt, I.W. (1871) Phil. Mag., 41, 447-454.

6. Rayleigh, Lord (1899) Phil. Mag., 47, 375-384.

7. Walker, J. (1989) Sci. Am., 260, 84-87.

8. Young, A.T. (1982) Phys. Today, 35, 42-48.

9. Berne, B.J.; Pecora, R. (1990) Dynamic Light Scattering. with applications to chemistry, biology and physics, Robert E. Krieger Publishing Company, Malabar (Florida), pag. 1-9.

10. Cummins, H.Z; Knable, N.; Yeh, Y. (1964) Phis, Rev Letts., 12, 150-154.

11. Pecora, R. (ed.) (1985) Dynamic Light Scattering: Applications of Photon Correlation Spectroscopy, Plenum Press, NY, pag. 1-6.

12. Jumel, K.; Browne, P.; Kennedy, I.F. (1992) em Harding. S.E.; Sattelle, D.B.; Bloomfield, V.A. (eds.), Laser Light Scattering in Biochemistry, The Royal Society of Chemistry, Cambridge, pag. 23-34.

13. Wyatt, P.J. (1992) em Harding, S.E.; Sattelle, D.B.; Bloomfield, V.A. (eds.), Laser Light Scattering in Biochemistry, The Royal Society of Chemistry, Cambridge, pag. 35-58.

14. McNeil-Watson, F.K.; Parker, A. (1992) em Harding. S.E.; Sattelle, D.B.; Bloomfield, V.A. (eds.), Laser Light Scattering in Biochemistry, The Royal Society of Chemistry, Cambridge, pag. 59-65.

15. Claes, P.; Dunford, M.; Kenney, A.; Vardy, P. (1992) em Harding, S.E.; Sattelle, D.B.; Bloomfield, V.A. (eds.), Laser Light Scattering in Biochemistry, The Royal Society of Chemistry, Cambridge, pag. 66-76.

16. Langley, K.H. (1992) em Harding, S.E.; Sattelle, D.B. Bloomfield, V.A. (eds.), Laser Light Scattering in Biochemistry, The Royal Society of Chemistry, Cambridge, pag. 151-160.

17. Weitz, D.A.; Pine, D.J. (1993) em Brown, W. (ed.), Dynamic Light Scattering - The Method and Some Applications, Oxford Science Publications, Oxford, pag. 652-720.
18. Pasternack, R.F.; Collings, P.J. (1995) Science, 269, 935939.

19. Philies, G.D.J. (1990) Anal. Chem., 62, 1049A-1057A.

20. Bloomfield, V.A. (1981) Ann. Rev. Biophys. Bioeng., 10 421-450.

21. Shepherd, I.W. (1975) Rep. Prog. Phys., 38, 565-620.

22. Flygare, W.H. (1977) Chem. Soc. Rev., 6, 109-137.

23. Ford, N.C.J. (1985) em Pecora R. (ed.), Dynamic Light Scattering: Applications of Photon Correlation Spectroscopy. Plenum Press, NY, pag. 7-58.

24. Marshall, A.G. (1978) Biophysical Chemistry: Principles, Techniques, and Applications, John Wiley \& Sons, NY, pag. 463-503.

25. Jelinek, Z.K. (1970) Particle Size Analysis, John Wiley \& Sons, NY, pag. $35-48$.

26. Casassa, E.F. (1989) em Brandrup, J.; Immergut, E.H. (eds.) Polymer Handbook, $3^{3}$ ed., John Wiley \& Sons, NY. pag. VII/485-492.

27. Weissberger, A.; Rossiter, B.W. (eds.) (1972) Techniques of Chemistry - Volume I: Physical Methods of Chemistry, John Wiley \& Sons, NY, pag. 75-101.

28. Schmitz, K.S. (1990) An Introduction to Light Scattering by Macromolecules, Academic Press, NY, pag. 1. 98.

29. Chu, B. (1991) Laser Light Scattering: Basic Principles and Practice, $2^{a}$ ed., Academic Press, NY, pag. 93-96.

30. Bloomfield, V.A. (1985) em Pecora R. (ed.), Dynamic Light Scattering: Applications of Photon Correlation Spectroscopy, Plenum Press, NY, pag. 363-406.

31. Pusey, P.N.; Tough, R.I.A. (1985) em Pecora R (ed.), Dynamic Light Scattering: Applications of Photon Correlation Spectroscopy, Plenum Press, NY, pag. 85-180.

32. Štépánek, P. (1993) em Brown, W. (ed.), Dynamic Ligh Scattering - The Method and Some Applications, Oxford Science Publications, Oxford, pag. 177-241.

33. Johnsen, R.M.; Brown, W. (1992) em Harding, S.E.; Sattelle, D.B.; Bloomfield, V.A. (eds.), Laser Light Scattering in Biochemistry, The Royal Society of Chemistry, Cambridge, pag. 77-91.

34. Provencher, S.W. (1992) em Harding, S.E.; Sattelle, D.B.; Bloomfield, V.A. (eds.), Laser Light Scattering in Bioche. mistry, The Royal Society of Chemistry, Cambridge, pag. 92 111.

35. Koppel, D.E. (1972) J. Chem. Phys., 57, 4814-4820.

36. Burchard, W. (1992) em Harding, S.E.; Sattelle, D.B. Bloomfield, V.A. (eds.), Laser Light Scattering in Biochemistry, The Royal Society of Chemistry, Cambridge, pag. 322.

37. Douglas, J.F.; Roovers, J.; Freed, K.F. (1990) Macromolecules, 23, 4168-4180. 